\title{
Streptococcus Dysgalactiae Subspecies Dysgalactiae Infection after Total Knee Arthroplasty: A Case Report
}

\author{
Man Jun Park, $\mathrm{MD}^{1}$, Il-Soo Eun, $\mathrm{MD}^{1}$, Chul-Young Jung, $\mathrm{MD}^{1}$, Young-Chul Ko, $\mathrm{MD}^{1}$, Young-June Kim, $\mathrm{MD}^{1}$, \\ Chang-kyu Kim, $\mathrm{MD}^{1}$ and Eun-Jin Kang, $\mathrm{MD}^{2}$ \\ Departments of ${ }^{1}$ Orthopedic Surgery, ${ }^{2}$ Internal Medicine, Busan Medical Center, Busan, Korea
}

\begin{abstract}
Streptococcus dysgalactiae subspecies dysgalactiae (SDSD), Lancefield group C streptococcus, is an animal pathogen which often causes pyogenic infection in domestic animals. Human infection by SDSD has been reported as a cellulitis on the upper arm, but a prosthetic joint infection caused by SDSD after total knee arthroplasty (TKA) has not yet been reported in the literature demonstrating that its clinical manifestation and management have not been well established. In this case report, we aimed to present a case of SDSD prosthetic joint infection after TKA, which was successfully treated by two-stage re-implantation with an application of antibiotic-impregnated cement spacer.
\end{abstract}

Key words: Total knee arthroplasty, Streptococcus dysgalactiae subsp, Dysgalactiae, Infection.

Streptococcus dysgalactiae (SD), a Lancefield group C streptococcus, can be broadly classified into Streptococcus dysgalactiae subspecies equisimilis (SDSE) and Streptococcus dysgalactiae subspecies dysgalactiae (SDSD) ${ }^{1)}$. SDSD is a-haemolytic and has been characterized as a pathogen that causes pyogenic infection and arthritis in animals. It has been rarely associated with human infections. The only reported case was a report of upper limb cellulitis in a foreign study ${ }^{2)}$. In particular, infection caused by SDSD following total joint arthoplasty (TJA) has never been reported. Thus, its clinical manifestation and treatment outcome have not been addressed in the literature. In this report, we present a case of prosthetic joint infection due to SDSD after total knee arthroplasty (TKA) that was treated successfully with two-

Received July 23, 2011; Revised (1st) September 8, 2011;

(2nd) November 6, 2011; Accepted December 4, 2011.

Correspondence to: Il-Soo Eun, MD.

Department of Orthopedic Surgery, Busan Medical Center, 359 World

Cup-daero, Yeonje-gu, Busan 611-072, Korea.

Tel: +82-51-607-2866, Fax: +82-51-607-2551

Email: dreun7@hanmail.net

This is an Open Access article distributed under the terms of the Creative Commons Attribution Non-Commercial License (http://creativecommons.org/licenses/by-nc/3.0/) which permits unrestricted non-commercial use, distribution, and reproduction in any medium, provided the original work is properly cited. stage re-implantation using an antibiotic-impregnated cement spacer with a review of relevant literature.

\section{Case Report}

A 61-year-old male patient presented to our institution with a complaint of pain in the right knee that had aggravated for the last three days. On the same knee, he underwent posterior cruciate ligament (PCL) reconstruction surgery due to trauma seven years prior and TKA due to rheumatoid arthritis four years previously. Although the patient suffered no difficulties with walking and performing daily living activities, he was admitted for three months in the recent year due to the worsening of the arthritis. He had been on methotrexate, one of the disease modifying antirheumatic drugs (DMARD) for one year. Pain, swelling, burning sensation, and limited range of motion were observed in the right knee during physical examination. The leukocyte count was $6,770 / \mathrm{mm}^{3}$, erythrocyte sedimentation rate was $46 \mathrm{~mm} / \mathrm{hr}$, and C-reactive protein was $44.55 \mathrm{mg} / \mathrm{L}$. Plain radiographs revealed radiolucencies at the bone cement interface (Fig. 1), which led us to perform joint aspiration with a suspicion of deep infection. About $50 \mathrm{~mL}$ of purulent exudate was aspirated and SDSD was isolated from the culture, so surgical intervention was conducted. Pyogenic exudate and acute synovitis were noted during surgery. Femoral and tibial components could be removed without difficulty due to severe osteolysis. We did not remove the interference screws inserted in the previous PCL 
reconstruction because the insertion site in the medial femoral condyle was not affected by the infection. In addition, the screws were so firmly fixated that removal appeared impossible without special techniques and bone loss. Infiltration of more than ten multinuclear leukocytes per high power field was observed in the frozen biopsy samples. Accordingly, we performed a thorough debridement of the synovial sheath and inserted a block type cement spacer impregnated with a combination of three antibiotics (gentamycin $1 \mathrm{~g}$, third generation cephalosporin $4 \mathrm{~g}$, and vancomycin $4 \mathrm{~g}$ ) to improve infection management (Fig. 2). A splint was applied to the knee in a $30^{\circ}$ flexion after
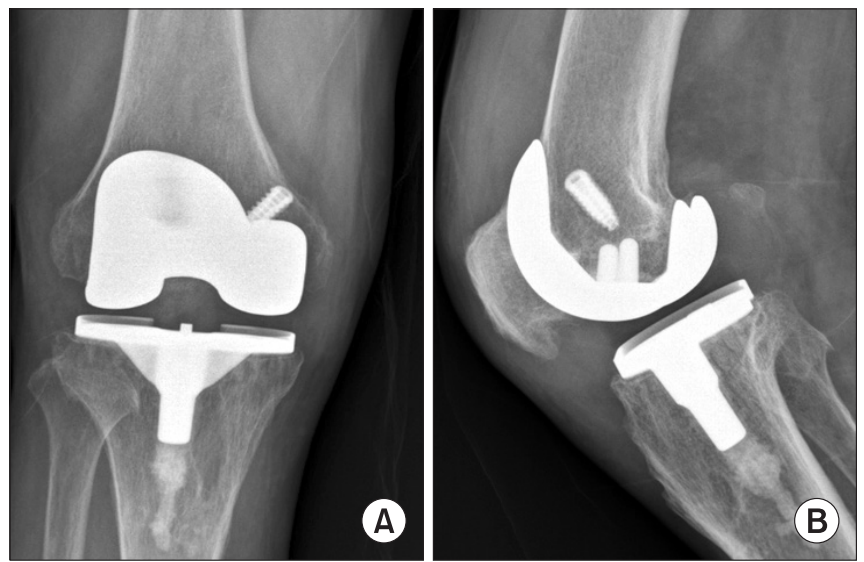

Fig. 1. Anteroposterior (A) and Lateral (B) views of an infected total knee arhtroplsty reveal mild radiolucencies at the bone cement interface of the medial and anterior aspects of the tibial and femoral component. surgery. Histology showed acute inflammation (Fig. 3) and the synovial fluid culture was positive for SDSD again. The SDSD cultured before and during surgery was resistant to oxacillin in the antibiotic sensitivity test. Accordingly, under the diagnosis of deep infection caused by SDSD resistant to oxacillin, vancomycin ( $2 \mathrm{~g}$ /day) was administered. In addition, third generation cephalosporin ( $2 \mathrm{~g} /$ day, ceftriaxone sodium) was used as a prophylactic agent to prevent other infections in the immunocompromised patient with an opportunistic infection. At seven weeks after surgery, the antibiotic treatment
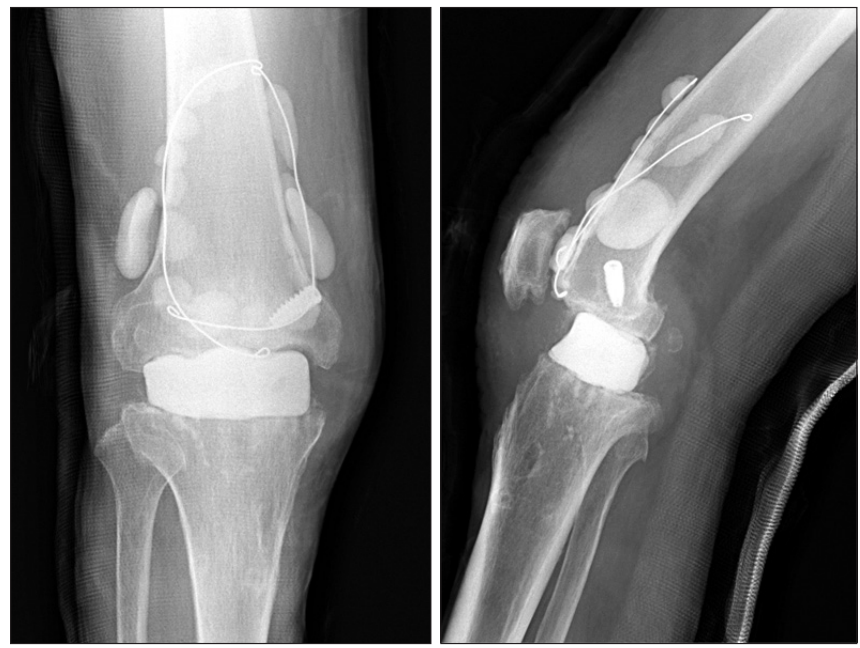

Fig. 2. After removal of the implants, antibiotic-cement block was inserted.
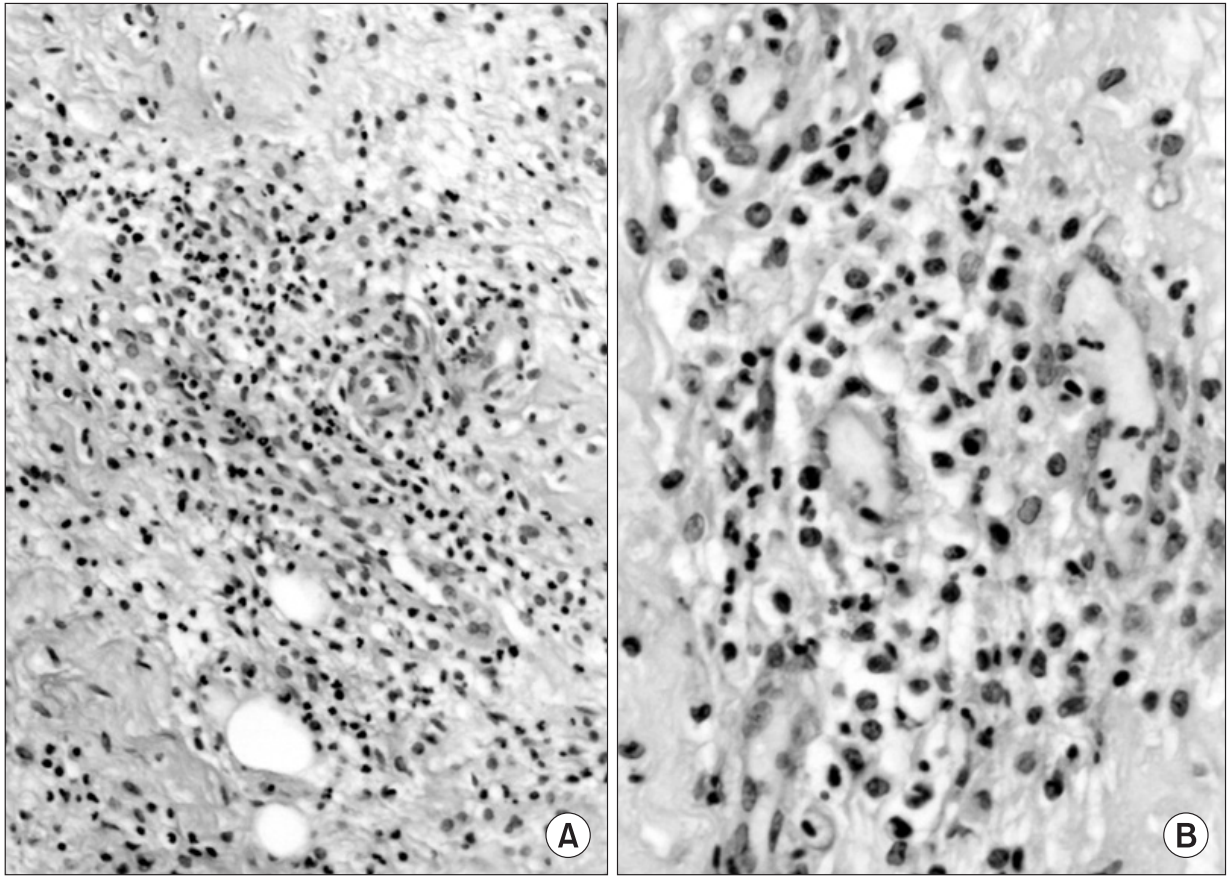

Fig. 3. Standard histology with hematoxyline-eosin staining contains acute granulation and inflammation. There are acute fibroblasts and neutrophils (H\&E, A: $\times 40, B$ : $\times 200$ ). 
was discontinued because the leukocyte count, erythrocyte sedimentation rate, and C-reactive protein levels were normal and the swelling, burning sensation, and flare improved. The infection did not recur in the following two weeks, so revision TKA was performed. Synovial exudate was not noted during surgery and infiltration of one multinuclear leukocyte in the high power field was observed in the frozen biopsy samples (Fig. 4). Postoperatively, the burning sensation and flare continued and the C-reactive protein level increased to $53.09 \mathrm{mg} / \mathrm{L}$. To prevent the recurrence of the SDSD infection and other infections that may be caused by the long-term hospitalization, vancomycin ( 2 $\mathrm{g} /$ day) and third generation cephalosporin ( $2 \mathrm{~g} /$ day, ceftriaxone sodium) were injected at the same time. The C-reactive protein was $23.88 \mathrm{mg} / \mathrm{L}$ at two weeks after surgery and $10.12 \mathrm{mg} / \mathrm{L}$ at
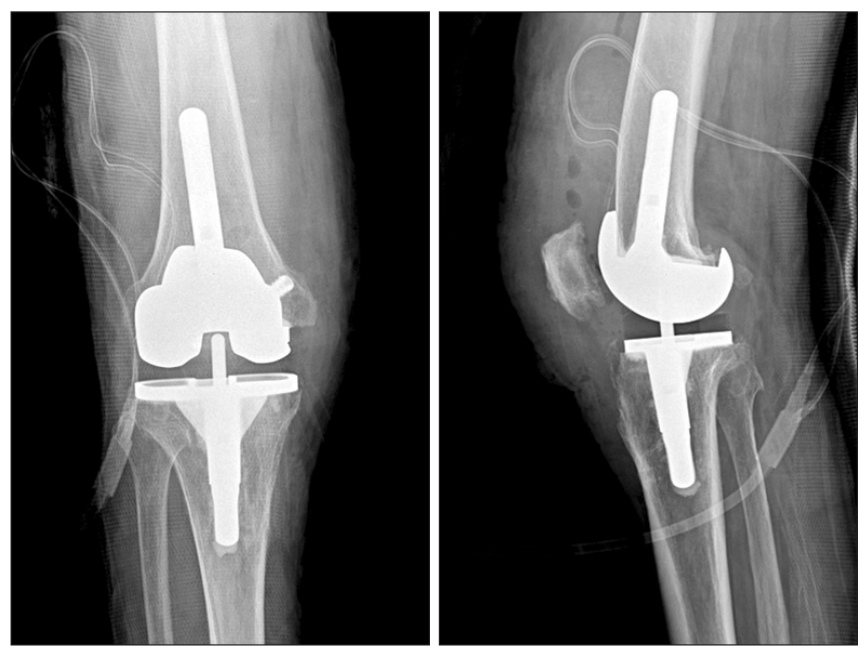

Fig. 4. Revision arthroplasty was performed as a second-stage procedure at 7 weeks after implant removal. four weeks after surgery and the symptoms improved. Therefore, the antibiotic treatment was replaced with oral administration of third generation cephalosporin (cefcapene pivoxil hydrochloride $300 \mathrm{mg} /$ day) for the following six weeks. Blood tests were performed every seven days, which showed no abnormal findings. Currently, at six months after surgery, no pain, burning sensation, and swelling have been observed. From four months after surgery, heterotopic ossification in the anterior aspect of the distal femur was observed on plain radiographs. However, magnetic resonance imaging has not revealed abnormal findings such as soft tissue inflammation (Fig. 5) and the range of motion has improved to $10^{\circ}$ in flexion contracture and $75^{\circ}$ in further flexion.

\section{Discussion}

Coagulase-negative Staphylococcus and Staphylococcus aureus have been known as the most common causative organisms of deep infection after TJA, followed by gram-negative bacteria, fungi, and mycobacterium tuberculosis. Specifically, streptococci are responsible for $7 \%$ of deep infection cases after TJA ${ }^{3)}$. Streptococci are mostly alpha or beta hemolytic and may become pathogenic in patients with immunological failure or malignant tumors. Lancefield classified streptococci from $\mathrm{A}$ to $\mathrm{V}$ depending on the antigen ${ }^{1)}$. Of these, $S$. pyogens (Lancefield group A) and $S$. agalactiae (Lancefield group B) have been frequently involved in infections in humans including pharyngitis, septic arthritis, and sepsis and recognized as an important pathogen of infections after TJA. On the other hand, Lancefield group C streptococci that may exist among the normal flora have rarely been described as a causative pathogen of infections after TJA. In 2000,
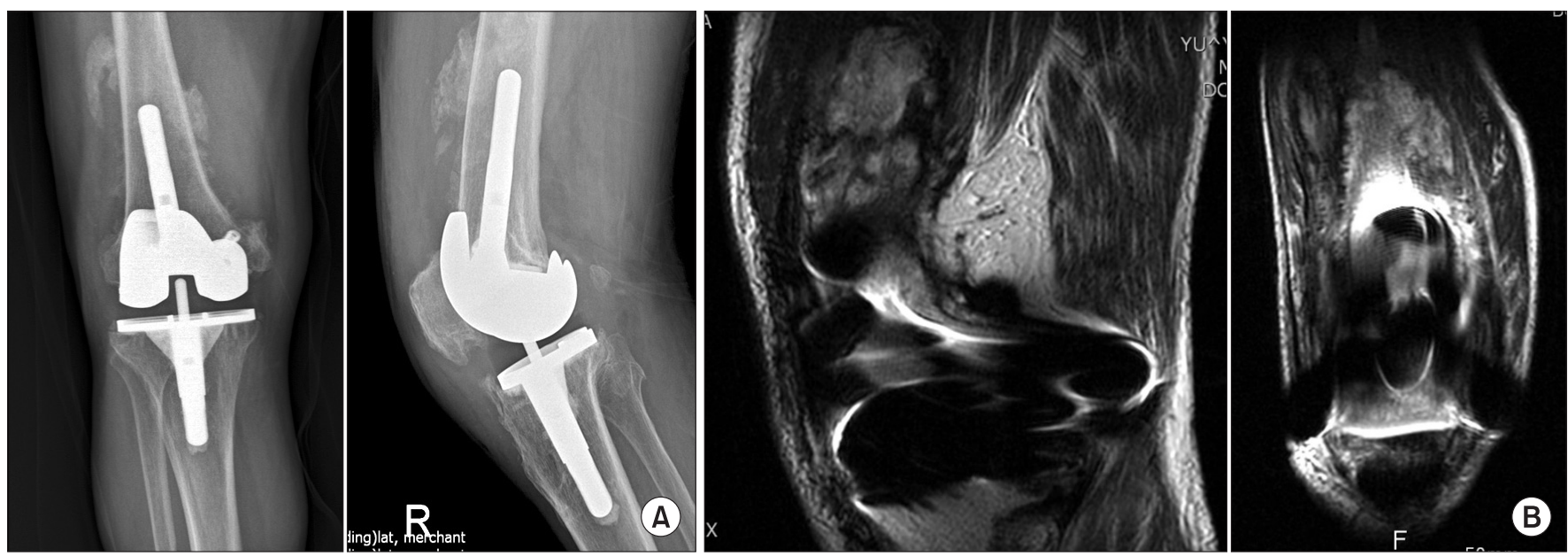

Fig. 5. Follow-up X-ray (A) and magnetic resonance imaging (B) show heterotrophic ossification around the distal femur. 
Kleshinski et al. ${ }^{4)}$ first reported a case of prosthetic joint infection caused by a Lancefield group C streptococcus, but it was not identified clearly whether SD was responsible for the infection because blood cultures were negative.

In 1996, Vandamme et al. ${ }^{5}$ subdivided SD into SDSE (a human pathogen) and SDSD (an animal pathogen) and reported that SD does not cause infection in humans because SDSE is a part of the normal human flora and SDSD is zoonotic ${ }^{6}$. However, SDSE has recently been identified as a beta hemolytic pathogen that can cause laryngopharyngitis and pneumonia in immunocompromised individuals and the elderly ${ }^{7}$. FernandezMartinez et al. ${ }^{8)}$ reported a case of septic arthritis in the knee suggesting that $\mathrm{SD}$ can cause harm to humans. Takahashi et al. ${ }^{9)}$ emphasized the risk of SDSE in the aging society where infections caused by SDSE are increasingly encountered in elderly patients. In the study by Koh et al. ${ }^{2}$, upper limb cellulitis was caused by SDSD in a patient who was injured while cleaning a fish and was treated successfully with antibiotics. They concluded that SDSD can cause human infections especially for individuals handling livestock and seafood. Our patient had no history of exposure to fish or animals, but he had been on a DMARD for a continuous period due to rheumatoid arthritis. Rheumatoid arthritis patients are 2.5 times more likely to have infections after TKA than other patients and the use of immunosuppressive agents such as DMARDs increases the risk of infection ${ }^{10)}$. Therefore, we believe that SDSD caused an opportunistic infection in our patient whose immune system was suppressed by rheumatoid arthritis medication.

Regarding the SD infection management, human infections caused by SDSD have not been documented in the literature. However, we believe the same treatment modalities for SDSEcaused infections can also be effective for SDSD-caused infections considering that there are no differences in the phenotypic characteristics between the SDSE strains and SDSD strains ${ }^{9)}$. Lancefield group C streptococci are normally treated with penicillin and SDSE frequently develops resistance to macrolide and quinolone, not to penicillin and cephalosporin".

The selection of antibiotics and duration of treatment may be controversial. We used vancomycin in our patient based on the culture tests in which SDSD was identified as the causative pathogen and the antibiotic sensitivity test. In addition, third generation cephalosporin was injected at the same time to prevent other infections in the immunocompromised patient with an opportunistic infection. After revision arthroplasty, antibiotics were administered continuously until symptoms were improved to prevent the recurrence of the SDSD infection because of the development of postoperative symptoms and blood test results and a second infection that may be caused by the long hospitalization

SDSD can cause cross infection from animals to humans, especially in immnocompromised patients and individuals in the fishing or animal industry. Human infection by SDSD after TKA is very rare and can be treated with two-stage re-implantation.

\section{References}

1. Hardie JM, Whiley RA. Classification and overview of the genera Streptococcus and Enterococcus. Soc Appl Bacteriol Symp Ser. 1997;26:1S-11S.

2. Koh TH, Sng LH, Yuen SM, Thomas CK, Tan PL, Tan SH, Wong NS. Streptococcal cellulitis following preparation of fresh raw seafood. Zoonoses Public Health. 2009;56:206-8.

3. Phillips JE, Crane TP, Noy M, Elliott TS, Grimer RJ. The incidence of deep prosthetic infections in a specialist orthopaedic hospital: a 15-year prospective survey. J Bone Joint Surg Br. 2006;88:943-8.

4. Kleshinski J, Georgiadis GM, Duggan JM. Group C streptococcal infection in a prosthetic joint. South Med J. 2000;93:1217-20.

5. Vandamme P, Pot B, Falsen E, Kersters K, Devriese LA. Taxonomic study of lancefield streptococcal groups $\mathrm{C}$, $\mathrm{G}$, and L (Streptococcus dysgalactiae) and proposal of S. dysgalactiae subsp. equisimilis subsp. nov. Int J Syst Bacteriol. 1996;46:774-81.

6. Facklam R. What happened to the streptococci: overview of taxonomic and nomenclature changes. Clin Microbiol Rev. 2002;15:613-30.

7. Efstratiou A. Pyogenic streptococci of Lancefield groups C and $\mathrm{G}$ as pathogens in man. Soc Appl Bacteriol Symp Ser. 1997;26:72S-9S.

8. Fernandez-Martinez AI, Pascual MR, Cimas D, Esteban J. Septic arthritis due to Streptococcus dysgalactiae ssp. equisimilis. Enferm Infecc Microbiol Clin. 2008;26:670-1.

9. Takahashi T, Ubukata K, Watanabe H. Invasive infection caused by Streptococcus dysgalactiae subsp. equisimilis: characteristics of strains and clinical features. J Infect Chemother. 2011;17:1-10.

10. Poss R, Thornhill TS, Ewald FC, Thomas WH, Batte NJ, Sledge CB. Factors influencing the incidence and outcome of infection following total joint arthroplasty. Clin Orthop Relat Res. 1984;(182):117-26. 